\title{
Analyse spatio-temporelle de la faune et des pressions anthropiques dans le ranch de Gibier de Nazinga au Burkina Faso
}

\author{
O. I. AMAHOWE ${ }^{1,2}$, M. OUEDRAOGO ${ }^{3}$ et O. T. LOUGBEGNON $^{4 *}$ \\ ${ }^{l}$ Institut International d'Ingénierie de l'Eau et de l'Environnement de Ouagadougou, Burkina Faso. \\ ${ }^{2}$ Centre National de Gestion des Réserves de Faune, Benin. \\ ${ }^{3}$ African Wildlife Foundation Bureau Afrique de l'Ouest Diapaga, Burkina Faso. \\ ${ }^{4}$ Université d'Abomey-Calavi, Benin. \\ *Auteur correspondant, E-mail: tlougbe@yahoo.fr
}

\section{RESUME}

Le ranch de Gibier de Nazinga au Burkina-Faso dispose d'un système de suivi écologique qui fournit une base de données permettant d'évaluer son potentiel faunique et d'analyser la distribution spatiale de la faune mammalienne et des indices de présence humaine. Ainsi, à partir de cette base de données, les estimations des effectifs et des densités animales de 2004 à 2009 ont été faites. Elles ont montré une instabilité pour la plupart des espèces animales. Les variations observées résultent des effets conjugués de l'exploitation cynégétique et de l'évolution naturelle corolaires de la forte pression humaine provenant des zones riveraines. L'analyse spatiale a révélé une exploitation orientée vers la partie centrale du ranch à la recherche des conditions optimales telles que l'eau et la quiétude. Les résultats obtenus sont d'un grand intérêt pour le plan de sauvegarde du ranch et pour la gestion cynégétique en vue d'un maintien de la diversité faunique et des services de l'écosystème rendus non seulement aux riverains mais également à l'Etat burkinabé.

(C) 2012 International Formulae Group. All rights reserved.

Mots clés: Faune, fluctuation spatio-temporelle, pressions anthropiques, ranch de Gibier de Nazinga, suivi écologique.

\section{INTRODUCTION}

L'importance de conserver la diversité biologique pour l'avenir de l'humanité est désormais reconnue au niveau mondial et largement médiatisée (Drouet-Hoguet, 2007).

En Afrique, plus que n'importe où au monde, la conservation de la faune sauvage est un enjeu crucial de société, car elle représente l'une des plus importantes richesses naturelles renouvelables de nombreux pays (PNGFAP, 2006). Au cours des dernières décennies, le phénomène d'érosion des populations de la grande faune mammalienne d'Afrique de l'Ouest a atteint un taux alarmant, et rien ne permet de croire que ce processus se ralentira dans un proche avenir (Cornelis, 2000).

Doumengue et al. (2001) ont démontré que les aires protégées constituent l'élément clé de toute stratégie de conservation de la biodiversité d'un pays ou d'une région. Au Burkina-Faso, la gestion de la faune intégrée aux multiples ressources forestières, intéresse toutes les couches sociales et particulièrement les populations rurales qui ont assuré pendant des siècles la conservation des espèces et de 
leurs habitats (PNGFAP, 2006). Cependant, le document de stratégie de réduction de la pauvreté a souligné que la perte de la biodiversité nécessite une attention particulière (World Bank, 2008). La dégradation et l'insuffisance des ressources naturelles font partie actuellement des problématiques du développement durable au Burkina-Faso. Dès lors, la faune sauvage, composante essentielle de la biodiversité nationale, devient une ressource stratégique pour tout le pays. Au vu des efforts entrepris dans le domaine de la gestion des ressources naturelles, le Burkina-Faso est considéré comme un pays réputé pour gérer la faune comme étant une ressource renouvelable (Vermeulen et al., 2007).

$\mathrm{Au}$ Burkina Faso, les aires protégées couvrent une superficie d'environ 2.545.500 ha soit à peu près $9,3 \%$ du territoire national (Dibloni, 2003). Le système national des aires protégées du Burkina Faso constitue un élément clé de la stratégie de conservation de la biodiversité. Le Ranch de Gibier de Nazinga (RGN) est la première expérience en Afrique francophone dans le domaine de la production faunique associant les communautés locales et la sécurisation de la biodiversité à travers une gestion durable (Ouédraogo, 2005). Il représente de ce fait un intérêt économique très important et une source de revenus diversifiée pour toute une série d'acteurs de conservation de la diversité biologique y compris les communautés locales (Bouché, 2004). Il a acquis dans la gestion de la faune une expérience qui fait de lui "un laboratoire naturel" (Yaméogo, 2005).

Le recensement de la faune est le tout premier outil requis pour la surveillance de l'évolution des populations animales, mais aussi pour la définition de stratégies adaptées aux objectifs de gestion de chaque aire protégée (Smallwood et Fitzhugh, 1995). Les données collectées permettent non seulement l'estimation de la faune, mais également l'analyse spatiale afin de déterminer les zones de concentration de la faune et des menaces auxquelles elle est sujette. Ceci permet d'obtenir des informations utiles pour l'efficacité des plans de sauvegarde des aires protégées.

Le présent travail vise 1'analyse de la dynamique spatiale de la grande faune et des menaces anthropiques auxquelles elle est sujette ces dernières années dans le Ranch de Nazinga. Ceci permettra de mieux orienter les politiques de gestions de cette réserve unique en Afrique de l'Ouest.

\section{MATERIEL ET METHODES \\ Milieu d'étude}

Créé en 1979 sur la forêt classée de Nazinga et élargi aux terroirs riverains connexes, le RGN est situé dans la partie méridionale du Burkina-Faso entre les latitudes $11^{\circ} 00^{\prime}$ et $11^{\circ} 18^{\prime}$, Nord et les longitudes $01^{\circ} 16^{\prime}$ et $01^{\circ} 43$ ' Ouest, avec une superficie d'environ 97.000 ha (Ouédraogo, 2005) (Figure 1). Il appartient à la zone soudanienne méridionale (Sivakumar et Gnoumou, 1987). Il est à $55 \mathrm{~km}$ de la ville de Pô et à environ $200 \mathrm{~km}$ de la capitale Ouagadougou. Le RGN s'étend sur sa plus grande partie sur la province du Nahouri (90\%) et une petite portion sur la province de Sissili (SOFRECO, 2003).

La hauteur moyenne annuelle des pluies calculée sur 30 ans (1978-2008) est de $925,98 \mathrm{~mm}$. La température moyenne annuelle dans les régions de Nazinga est de $27,80{ }^{\circ} \mathrm{C}$. Les valeurs fortes de températures moyennes sont enregistrées en mars-avril (autour de 31 $\left.{ }^{\circ} \mathrm{C}\right)$ et les faibles valeurs en août $\left(25,29{ }^{\circ} \mathrm{C}\right)$. Le Ranch de Gibier de Nazinga est traversé principalement par la rivière Sissili. Les cours d'eau secondaires sont: le Dawévélé et le Nazinga.

L'écologie des herbivores est intimement liée à la végétation, qui fournit entre autres leurs ressources alimentaires, de l'ombre, un abri contre les prédateurs (Rosenweig, 1991; Mbuma et Sinclair, 1994; Chardonnet et al. 1995). La végétation est essentiellement constituée d'une mosaïque de savanes et de forêts galeries. Dekker (1985) révèle une forte proportion de savanes claires 
(47,4\%) à Vitellaria paradoxa, Terminalia spp., Acacia dudgeoni, Gardenia erubescens, Pteleopsis suberosa; 25,4\% des savanes arborées à Afzelia africana, Anogeissus leiocarpa et Lannea acida; 15,6\% de la savane à Detarium microcarpum. La strate herbacée est dominée par des graminées pérennes telles que Andropogon ascinodis, Hyparrhenia spp et Schizachyrium sanguineum (Delvingt et Vermeulen, 2007). Sur les sols plus profonds, la végétation change en savane boisée à Hyparrhenia smithiana, Detarium microcarpum, Afzelia africana, Burkea africana, Pteleopsis suberosa, Acacia dudgeoni, Isoberlinia doka, Anogeissus leiocarpa et Vitellaria paradoxa (Dekker, 1985). La faune de Nazinga est représentée par les espèces animales de la savane soudanienne. Son principal attrait est sa grande population d'éléphants. Il existe actuellement plus de dix (10) espèces d'ongulés d'une grande valeur cynégétique et touristique. En plus de l'éléphant, les mammifères les plus visibles sont composés de: buffle (Syncerus caffer brachyceros), hippotrague (Hippotragus equinus koba), bubale (Alcelaphus buselaphus), Cobe de Buffon (Kobus kob kob), cobe defassa (Kobus ellipsiprymnus defassa), cobe redunca (Redunca redunca), guib harnaché (Tragelaphus scriptus scriptus), céphalophe de grimm (sylvicapra grimmia), céphalophe à flancs roux (Cephalophus rufilatus), ourébi (Ourebia ourebi), phacochère (Phacochoerus africanus).

\section{Méthode d'inventaire de la faune et des indices de présence humaine}

La technique utilisée pour le dénombrement de la faune est basée sur la définition de transects à largeur équidistante de 1400 m. Elle a été déjà utilisée avec succès par beaucoup d'autres auteurs ayant travaillé sur les écosystèmes de savanes en Afrique (Van Lavierien et Bosch, 1977; Cornelis, 2000; Belemsobgo, 2000; Ouédraogo, 2005). Elle est basée sur la relation entre la distance perpendiculaire d'un animal ou d'un troupeau à la ligne de transect et la probabilité de le détecter. Cette relation se traduit par la fonction de détection $\mathrm{g}(\mathrm{x})$ d'un objet à une distance perpendiculaire $\mathrm{x}$ du transect. Ici, l'animal ou le troupeau représente l'objet. Selon Buckland et al. (2004), la méthode de "Distance sampling" fonctionne sur les hypothèses suivantes : (i) la probabilité qu'un animal ou un troupeau situé sur la ligne du transect soit détecté est égale à $1(\mathrm{~g}(0)=1)$. Cette probabilité décroît lorsque la distance d'observation de l'animal au transect augmente; (ii) les animaux sont détectés à leur position initiale, ils ne doivent pas se déplacer en réponse à l'observateur avant d'être détectés. Si les animaux fuient avant d'être détectés par l'observateur, la distribution des distances perpendiculaires ne reflètera pas la forme de la fonction de détection; (iii) les distances sont estimées avec précision. Lorsqu'il y a des erreurs dans la mesure des distances, elles peuvent induire des biais dans l'estimation des densités; (iv) les détections des animaux sont indépendantes. La dépendance peut arriver lorsque les animaux sont regroupés en «cluster» sur la surface échantillonnée.

Le dispositif d'inventaire total est composé de 79 transects d'une longueur totale d'environ $692 \mathrm{~km}$ répartis en sept (07) unités. Le réseau de piste disponible est utilisé pour une meilleure organisation des inventaires. Les transects sont disposés tous les 1,4 km dans la direction Sud-Nord (Figure 2). L'azimut de marche sur les transects est de $6^{\circ}$. Les équipes prennent les coordonnées géographiques des points d'observation. De plus, pour chaque troupeau/animal observé, les informations suivantes sont notées : le nom de l'espèce, les coordonnées d'observation des animaux, l'angle $(\alpha)$ de vue est l'angle entre la ligne du transect et la ligne joignant l'observateur à l'animal, la distance radiale (r) est la distance entre l'observateur et la position initiale de l'animal. La distance perpendiculaire $(\mathrm{X})$ de l'animal par rapport au transect est calculée par la formule $X=$ $r^{*} \sin (\alpha)$ avec $\alpha=$ angle d'observation de 
l'animal à partir de la ligne de marche angle vu-azimut. Les transects sont équidistants de $1400 \mathrm{~m}$. La méthode ainsi décrite permet une estimation des effectifs, la présentation de la distribution spatiale des espèces étudiées ainsi que celle des indices de présence humaine.

\section{Période de prospection et nombre de mission}

Le dispositif d'inventaire décrit cidessus a été répété durant 6 inventaires successifs dans le RGN entre 2004 et 2009. Ceci justifie que les données utilisées pour la présente étude soient issues d'une base de données d'un système de biomonitoring du RGN.

\section{Analyse des données \\ Estimation des densités et des effectifs de faune}

La densité et les effectifs des animaux ont été calculés à l'aide du logiciel Distance (Laake et al., 1994). Ainsi, la densité est déduite de la probabilité de détecter un animal sur une superficie échantillonnée. La formule générale permettant d'estimer cette densité est : $\mathrm{D}=\mathrm{N} / \mathrm{a}$ avec $\mathbf{N}=$ nombre d'animaux observés et a la surface couverte par les transects. $a=2 \mathrm{Lw}$ avec $\mathrm{L}$ la longueur totale des transects inventoriés et w la demi largeur de la bande. Ainsi, la densité $D=N / 2 L w$. Le nombre d'animaux observés correspond au nombre d'animaux présents $\mathrm{N}$ multiplié par la probabilité de détection $\mathrm{P}_{\mathrm{a}}$.

Ensuite, a suivi la modélisation de la fonction de détection à partir d'une série de fonction ( « Key fonction» ou $\mathrm{k}(\mathrm{y})$ ) et des termes d'ajustement « séries expansion » ou « series (y) ». La fonction de détection est modélisée selon la formule : $\mathrm{g}(\mathrm{x})=\mathrm{key}(\mathrm{y})$ $[1+\operatorname{serie}(\mathrm{y})]$ et son estimation s'est faite à l'aide des données de distance en supposant que les hypothèses citées ci-dessus sont respectées.

A partir du test des fonctions mathématiques (test $\mathrm{Chi}^{2}$ ) et du test d'AIC (Critère d'Information d'Akaike), le modèle qui se rapproche le plus de la distribution des distances perpendiculaires a été choisi. Les densités et les effectifs des espèces animales ont été directement calculés avec les intervalles de confiance correspondant.

\section{Calcul des indices kilométriques d'abondance (IKA) et Indices kilométriques de Contact (IKC)}

L'IKA est un paramètre intéressant pour la définition des quotas de chasse à défaut de disposer des effectifs de chaque espèce animale dans la réserve faunique, même s'il ne renseigne pas sur les densités animales. Il offre des données brutes sans intervalle de confiance et donc plus facile à interpréter. Le IKA a été calculé pour toutes les espèces y compris celles dont le nombre d'observations était insuffisant. C'est le rapport du nombre d'individus observés sur la distance parcourue exprimée en kilomètres. Il se calcule pour une espèce ou pour l'ensemble des espèces dans une zone. Effectué régulièrement dans la même zone et dans les mêmes conditions, il est un bon indicateur de la tendance des populations animales, car il permet de savoir si la population augmente, diminue ou stagne (Tsakem, 2006).

Par contre, l'indice kilométrique de contact renseigne sur la fréquence d'observation par $\mathrm{km}$ d'une espèce. Il peut être également calculé pour un ensemble d'espèces.

\section{Analyse spatiale \\ Distribution spatiale de la faune}

La distribution spatiale des espèces au sein du ranch a été testée par le calcul de l'indice de Blackman $\mathbf{I}_{\mathbf{B}} . \mathbf{I}_{\mathbf{B}}=\delta^{2} / \mu$ avec $\delta^{2}$ la variance de l'échantillon et $\mu$ la moyenne. Trois types de répartitions sont possibles : (i) $\mathbf{I}_{\mathbf{B}}=1$, la répartition est poissonnière; (ii) $\mathbf{I}_{\mathbf{B}}$ $<1$, la répartition est régulière ; (iii) $\mathbf{I}_{\mathbf{B}}>1$, la répartition est agrégative. Trois périodes (p1, p2 et p3) correspondant respectivement à 2004-2005, 2006-2007, 2008-2009 ont été considérées pour le calcul de la distribution spatiale globale de la faune. 


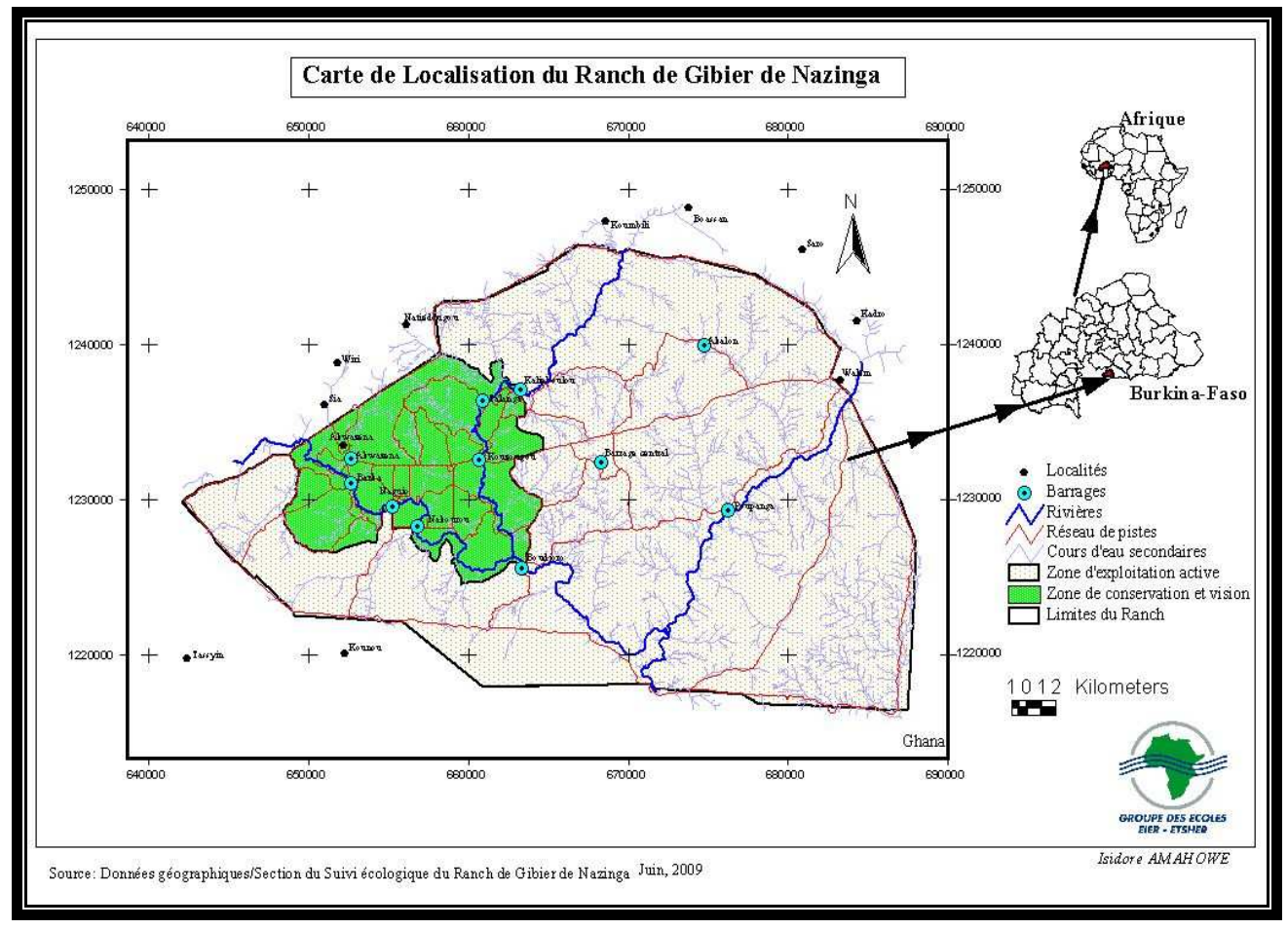

Figure 1 : Carte de situation du Ranch de Gibier de Nazinga en Afrique et au Burkina Faso.

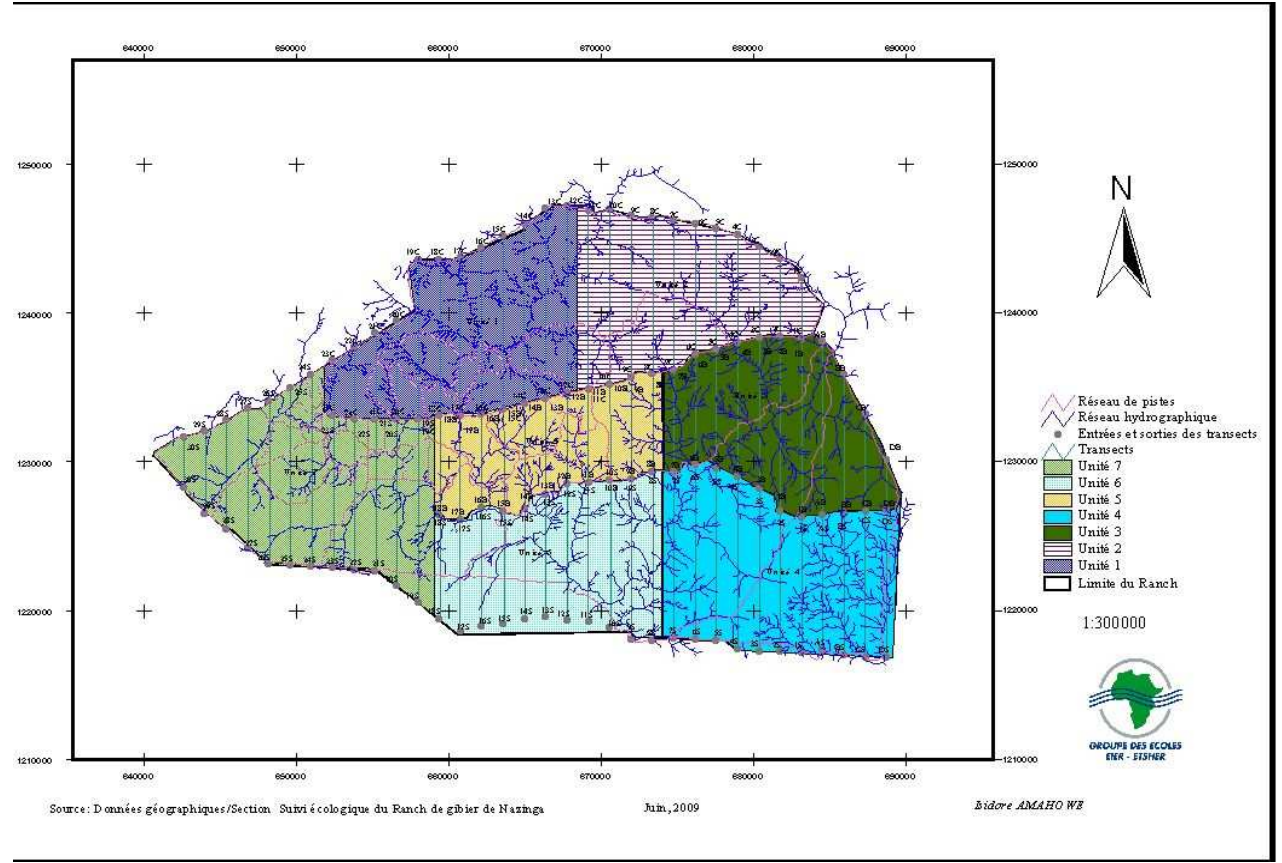

Figure 2 : Carte des transects d'inventaire. 
AMAHOWE O. I. et al. / Int. J. Biol. Chem. Sci. 6(2): 613-627, 2012

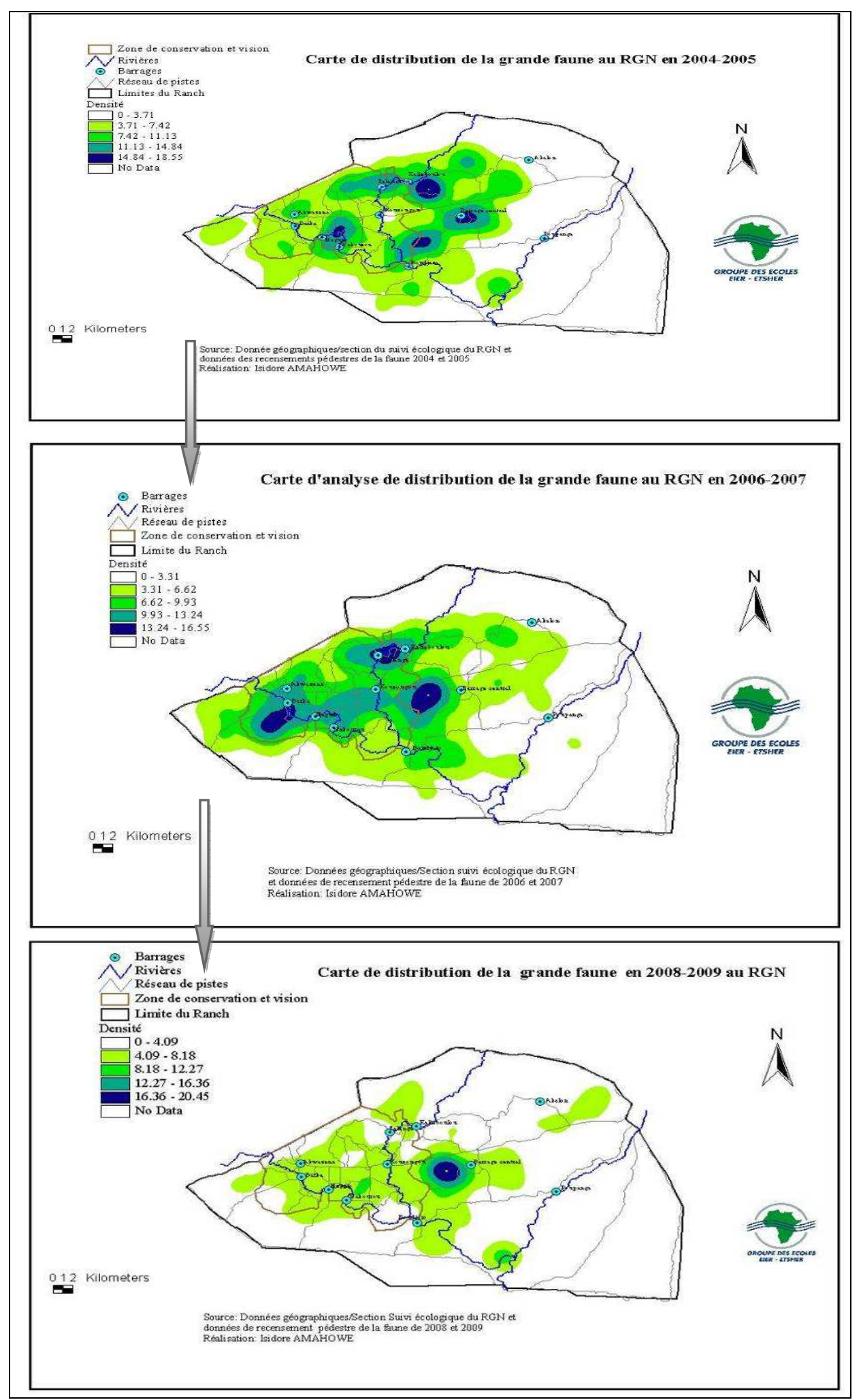

Figure 3: Distribution spatiale de la grande faune au Ranch de Gibier de Nazinga entre 2004 et 2009 . 
AMAHOWE O. I. et al. / Int. J. Biol. Chem. Sci. 6(2): 613-627, 2012

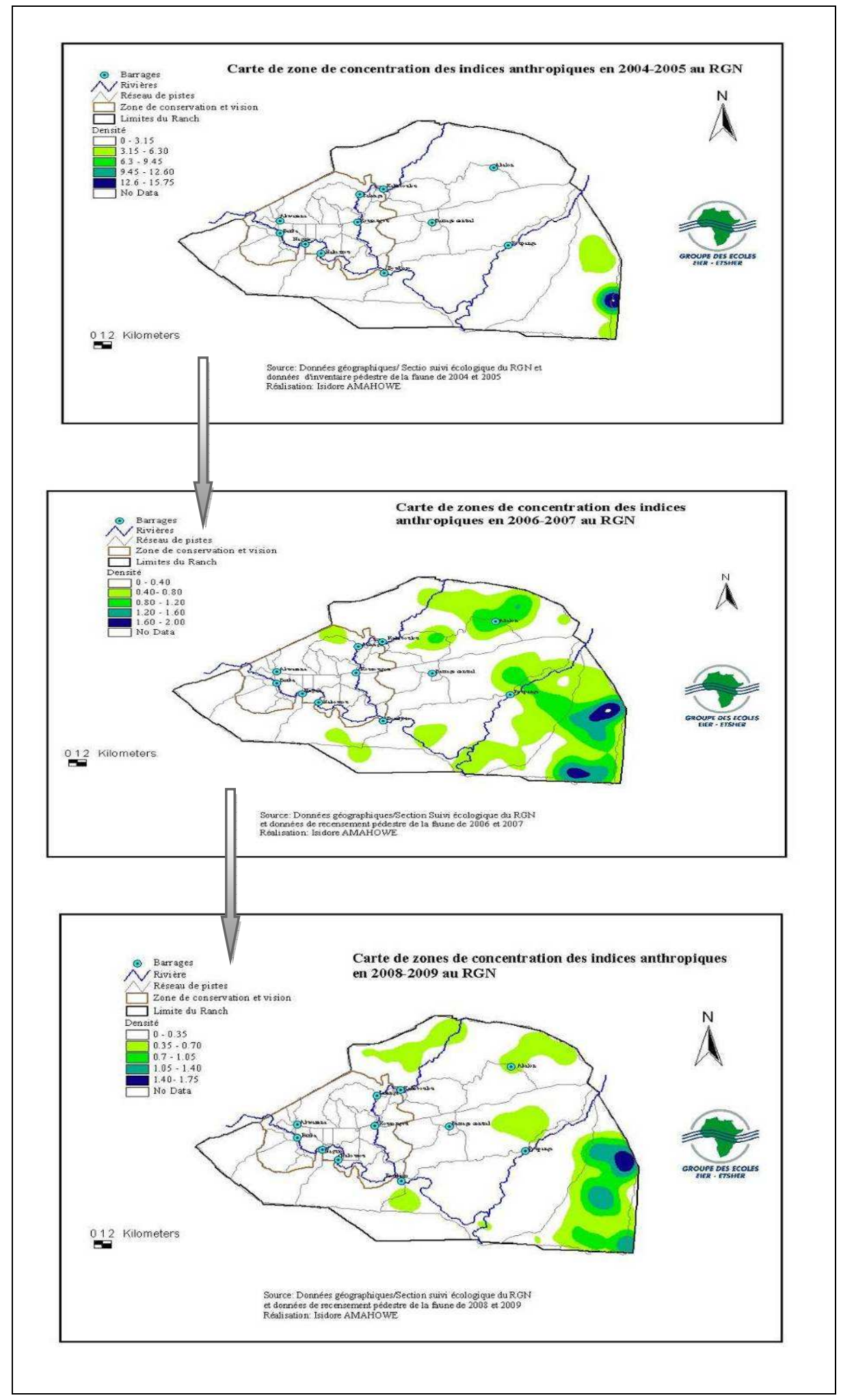

Figure 4: Distribution spatiale des indices anthropiques de 2004 à 2009 au RGN. 


\section{Estimation de la densité de Kernel}

La distribution spatiale de la faune a été réalisée sur l'ensemble des observations faites sur les espèces les plus rencontrées au RGN car elles seules permettent de remarquer facilement les variations affectant leurs abondances ou leurs réactions vis-à-vis des pressions anthropiques. Ces espèces sont le bubale (Alcelaphus buselaphus major), le koba (Hippotragus equinus koba), le waterbuck (Kobus ellipsiprymnus defassa), le phacochère (Phacochoerus africanus), le guib harnaché (Tragelaphus scriptus) et le céphalophe de grimm (Sylvicapra grimmia).

Les densités du buffle (Syncerus caffer) et de l'éléphant (Loxodonta. africana) n'ont pas été évaluées parce que les effectifs de ces deux espèces sont trop faibles et donc sont inadéquates à être soumis à la méthode de "Distance sampling".

Il s'agit de l'estimation de densité de Kernel (Worton, 1989; Cornelis, 2000) qui permet de déterminer les noyaux de concentration animale. Elle est une estimation non paramétrique plus utilisée pour la détermination du domaine vital des espèces (Worton, 1989; Laver, 2005) ou en criminologie (Brimicombe, 2007). Ainsi, les données des observations faites sur les six (06) inventaires de 2004 à 2009 ont été analysées dans le logiciel Arc View 3.2 avec les extensions Spatial analyst 2.0 et Animal Movement Extension. Les données de deux années successives ont été poolées afin d'obtenir le minimum de points d'observation exigé par la méthode (Brian, 2007) et tenant compte du fait qu'en absence d'une perturbation écologique remarquable, les animaux changent très peu de comportement spatial entre deux périodes relativement proches. De plus, à l'instar de Cornelis (2000) sur les mêmes transects, un rayon d'analyse constant de $3.000 \mathrm{~m}$ est employé afin d'intégrer les observations effectuées autour des transects voisins étant entendu que l'équidistance entre les transects est de 1.400 $\mathrm{m}$. Le pixel défini est $50 \mathrm{~m} \times 50 \mathrm{~m}$.

\section{RESULTATS \\ Evolution des populations de quelques espèces de grands mammifères au RGN Bubale}

La population des bubales a connu de façon générale une diminution de 2004 à 2009. Entre 2004 et 2006, les effectifs sont restés presque stables et ont oscillé entre 3.502 et 3.571 individus, avant de chuter à 2.414 individus en 2008, pour reprendre légèrement en 2009 avec 2.711 individus. Les densités suivent pratiquement la même tendance que celle des effectifs : elle diminue de 3,59 à 3,66 individus $/ \mathrm{km}^{2}$ entre 2004 à 2005, puis diminue de façon drastique à 2,16 individus $/ \mathrm{km}^{2}$ en 2007. Elle augmente de nouveau pour atteindre 2,78 individus $/ \mathrm{km}^{2}$ en 2009.

\section{Céphalophe de grimm}

Cette espèce s'observe faiblement au RGN. Sur les six recensements au RGN, seulement deux ont permis d'avoir au moins 30 observations. Les effectifs sont respectivement 559 et 670 individus pour les recensements de 2004 et 2007, puis les densités sont très faibles : 0,57 individus $/ \mathrm{km}^{2}$ en 2004 et 0,68 individus $/ \mathrm{km}^{2}$ en 2007.

\section{Hippotragues}

Les effectifs d'hippotragues ont augmenté de 2.866 à 3.839 individus de la période allant de 2004 à 2007, puis régressé à 2.211 en 2008 avant de croitre de nouveau à 9.304 individus en 2009. La densité augmente de 2,94 individus $/ \mathrm{km}^{2}$ à 3,93 individus $/ \mathrm{km}^{2}$ entre 2004 et 2007. Ensuite, elle diminue à 2,27 individus $/ \mathrm{km}^{2}$ en 2008, avant d'augmenter légèrement à 2,28 individus $/ \mathrm{k} \mathrm{m}^{2}$ en 2009.

\section{Guib harnaché}

Les effectifs de guib harnaché ont diminué de 1.719 individus en 2004 à 564 en 2006, puis augmenté à 896 individus en 2007. Dans le même temps, la densité a diminué de 1,76 à 0,57 individus $/ \mathrm{km}^{2}$ en 2006 avant d'augmenter à 0,92 individus $/ \mathrm{km}^{2}$ en 2007. En 2009 la densité de guib harnaché est de 0,7314 ind $/ \mathrm{km}^{2}$. Lors du recensement de 2008, le guib harnaché a été rarement observé, ce qui n'a 
pas permis une estimation de la densité par le programme Distance. En 2009, l'effectif du guib harnaché a été estimé à 713 individus au RGN avec une densité de 0,73 individus $/ \mathrm{km}^{2}$. Les densités suivent la même tendance que les effectifs avec $1,76 \mathrm{ind} / \mathrm{km}^{2}$ en 2004. Elle a diminué à $0,57 \mathrm{ind} / \mathrm{km}^{2}$ en 2006 puis augmenté à 0,92 ind $/ \mathrm{km}^{2}$ en 2006.

\section{Phacochère}

Les effectifs des phacochères diminuent de 7.081 à 3.287 entre 2004 et 2007, puis augmentent en 2008 à 4.224 individus. Mais on a constaté une diminution dramatique des effectifs en 2009 à 1.913 individus. Les densités de phacochère diminuent de 7,27 ind $/ \mathrm{km}^{2}$ en 2004 à 1,96 ind $/ \mathrm{km}^{2}$ en 2009.

\section{Waterbuck}

Les effectifs de Waterbuck ont varié sur les six (06) inventaires entre 2.812 et 973 individus. De 973 individus en 2008, l'effectif de Waterbuck a augmenté brusquement à 2.812 avec un large intervalle de confiance en 2009. La densité de waterbuck varie entre 1 et $2,88 \mathrm{ind} / \mathrm{km}^{2}$ et présente la même tendance que les effectifs.

Indices kilométriques d'Abondance (IKA)

Le Tableau 1 montre les tendances des IKA par espèce de grands mammifères recensés. Les valeurs moyennes des IKA de 2004 à 2009 sont élevées pour les espèces comme: koba $(0,57 \mathrm{ind} / \mathrm{km})$, bubale $(0,52$ ind $/ \mathrm{km})$, phacochère $(0,34 \mathrm{ind} / \mathrm{km})$, éléphant $(0,30 \mathrm{ind} / \mathrm{km})$ et babouin $(0,21 \mathrm{ind} / \mathrm{km})$. Dans la même période, les IKA sont restés faibles pour le buffle $(0,07 \mathrm{ind} / \mathrm{km})$, le guib harnaché (0,06 ind $/ \mathrm{km})$, l'ourébi $(0,05 \mathrm{ind} / \mathrm{km})$, le céphalophe de grimm $(0,04 \mathrm{ind} / \mathrm{km})$, le patas $(0,03 \mathrm{ind} / \mathrm{km})$, le cobe de buffon $(0,01$ ind $/ \mathrm{km})$, le redunca $(0,007 \mathrm{ind} / \mathrm{km})$ et le vervet $(0,01 \mathrm{ind} / \mathrm{km})$. Les carnivores en général sont très peu observés et présentent par conséquent des IKA presque nuls. Les IKC moyen sont dans l'ensemble très faibles. Les valeurs les plus élevées sont observées chez le koba $(0,1$ cont $/ \mathrm{km})$, le phacochère $(0,1$ cont $/ \mathrm{km})$ puis le bubale $(0,08 \mathrm{cont} / \mathrm{km})$.

\section{Distribution spatiale globale Indice de Blackman (IB)}

La distribution spatiale globale de la grande faune au RGN pendant chacune des périodes est agrégative (Indice de Blackman IB $>1$ ). Le Tableau 2 présente les résultats du test de la distribution de la grande faune au RGN sur les trois périodes (p1, p2 et p3). Les indices varient entre 5,85 et 7,44 sur les trois périodes. La distribution de la faune au RGN est agrégative pour chacune des périodes et ne montre pas une différence significative d'une période à l'autre.

\section{Analyse globale de la faune par la méthode de Kernel}

La distribution globale de la faune de 2004 à 2009 montre une variation de la densité de kernel de 0 à 18,55 ind $/ \mathrm{km}^{2}$ en 2004-2005, de 0 à $16,55 \mathrm{ind} / \mathrm{km}^{2}$ en 20062007, puis de 0 à 20,45 ind $/ \mathrm{km}^{2}$ en 2008-2009 (Figure 3).

En p1 on observe quatre noyaux de concentration de la faune (zone de densité d5) couvrant une superficie de $11,98 \mathrm{~km}^{2}$. Un noyau est situé au Nord-Est de Kaliéboulou, un deuxième au bord du barrage central, un troisième un peu au Nord-Est de Naguio, puis un quatrième entre les limites de la zone de conservation vision et la zone d'exploitation active, isolé des barrages. En p2, on note trois noyaux de concentration sur une superficie totale de $18,05 \mathrm{~km}^{2}$. Un noyau est observé au niveau de Talanga, un second au Sud de Barka et le troisième à l'Ouest du barrage central. Mais en p3, la faune s'est retirée sur un petit noyau de 4,8 km² à l'Ouest du barrage central, proche des limites de la zone de conservation et vision.

En général, les populations animales se sont retirées vers la partie Centre-Est du Ranch où elles exploitent mieux le milieu. Les populations fauniques ont réduit progressivement leur espace de vie de 44,14 à 26,13\% entre p1 et p3 (Tableau 3). En effet, on remarque à travers ce Tableau 3 que les densités occupées par la grande faune par zone ont considérablement diminué surtout de 2004 à 2007. Ceci traduit forcément une 
réaction à une agression ou intempérie extérieure.

\section{Indices anthropiques}

L'analyse spatiale des indices anthropiques sur l'aire du Ranch montre une variation de densité locale de 0 à 15,75 ind $/ \mathrm{km}^{2}$ en période $\mathrm{p} 1$, de 0 à $2 \mathrm{ind} / \mathrm{km}^{2}$ en période $\mathrm{p} 2$, puis de 0 à $1,75 \mathrm{ind} / \mathrm{km}^{2}$ en période p3 (Figure 4). En p1, les indices anthropiques occupent $3,4 \%$ de la superficie $\mathrm{du}$ ranch avec un seul noyau de forte concentration de densité d5 sur $2,84 \mathrm{~km}^{2}$. Le noyau est situé dans la partie Sud-Est du Ranch.

En p2, les pressions anthropiques se répartissent sur $32,35 \%$ de la superficie du Ranch avec deux noyaux de densité d5 sur $8,52 \mathrm{~km}^{2}$. Un noyau est situé à proximité de la base Sud du Ranch et le second plus étalé vers Sud-Est. A cette période, les indices anthropiques sont répartis sur une superficie importante (soit $32,35 \%$ de la superficie totale du Ranch).

En p3, les indices anthropiques sont compactés en un seul noyau toujours dans la partie Sud-Est, vers la périphérie du Ranch. Les indices anthropiques occupent au total $19,52 \%$ de la superficie du Ranch.

De façon générale, la partie incluant la périphérie Est et le Sud-Est du RGN vers la frontière ghanéenne subit de fortes agressions anthropiques.

\section{DISCUSSION}

\section{Analyse des tendances d'évolution des populations fauniques}

La comparaison des résultats de l'effectif des animaux durant la période de recensement de ce travail à ceux précédemment effectués dans le RGN par O’Donoghue (1986), et Cornelis (2000), fait remarquer que les effectifs de bubale sont relativement élevés de 2004 à 2009. On observe presque une stabilité des effectifs de bubale entre 2004 et 2006, suivie d'une baisse en 2007, puis une nouvelle reprise à partir de
2008. Les mouvements de troupeaux d'animaux entre le RGN, la forêt classée de Sissili et le Park Kaboré Tambi qui sont des aires connexes, pourrait expliquer cette instabilité des effectifs. En effet, il existe des couloirs de migrations pour la faune qui permettent des flux d'échanges de gènes entre le RGN et ces aires classées connexes.

Les populations de koba n'ont pas trop varié (1157 à 3788 individus) de la période allant de 1985 à 2008, mais il faudra souligner une hausse inhabituelle des effectifs en 2009. Ceci tient certainement de l'afflux des populations venant d'autres zones connexes.

Les estimations d'effectif et de densité ont été effectuées pour le waterbuck par la méthode de transect linéaire au RGN seulement de 2004 à 2009 en raison de l'insuffisance d'observations souvent constatée avant cette période. Ce qui fait penser que le waterbuck retrouve progressivement les conditions écologiquement favorables pour sa survie et son expansion au RGN. Mais il a été également constaté à l'instar de koba, une hausse inhabituelle des effectifs de waterbuck en 2009. Ces surestimations constatées seraient dues aux imprécisions lors de l'estimation des distances d'observation et au biais lors de l'estimation des effectifs des troupeaux. Des biais peuvent être enregistrés lors des estimations de distance au télémètre, lorsque les rayons fixent des objets autres que les animaux détectés sont en deçà.

S'agissant du céphalophe de grimm, sa population a diminué progressivement de 1985 à 1996, puis s'est accrue de nouveau sur deux ans avant de rechuter jusqu'en 2004. Après cette période, l'espèce s'est raréfiée et a pu être estimée seulement en 2007, faute d'observation suffisante. Cela est certainement dû aux mœurs de cette espèce qui est très craintive et ne supporte pas trop les activités anthropiques telles que la chasse, le tourisme de vision qui sont les plus pratiquées dans la période des inventaires. 
AMAHOWE O. I. et al. / Int. J. Biol. Chem. Sci. 6(2): 613-627, 2012

Tableau 1 : Tendances des IKA et IKC par espèce de grands mammifères recensés.

\begin{tabular}{|c|c|c|c|c|c|c|c|c|c|c|c|c|c|c|}
\hline \multirow[t]{2}{*}{ Espèces } & \multicolumn{2}{|c|}{2004} & \multicolumn{2}{|c|}{2005} & \multicolumn{2}{|c|}{2006} & \multicolumn{2}{|c|}{2007} & \multicolumn{2}{|c|}{2008} & \multicolumn{2}{|c|}{2009} & \multicolumn{2}{|c|}{ MOYENNE } \\
\hline & IKC & IKA & IKC & IKA & IKC & IKA & IKC & IKA & IKC & IKA & IKC & IKA & IKC & IKA \\
\hline Babouin & 0,039 & 0,238 & 0,036 & 0,219 & 0,04 & 0,206 & 0,042 & 0,23 & 0,02 & 0,166 & 0,039 & 0,208 & 0,036 & 0,211 \\
\hline Bubale & 0,081 & 0,624 & 0,092 & 0,671 & 0,108 & 0,559 & 0,091 & 0,409 & 0,081 & 0,452 & 0,061 & 0,445 & 0,086 & 0,527 \\
\hline Buffle & 0,004 & 0,081 & 0,009 & 0,102 & 0,004 & 0,046 & 0,007 & 0,128 & 0,003 & 0,032 & 0,004 & 0,059 & 0,005 & 0,075 \\
\hline Céphalophe de grimm & 0,053 & 0,059 & 0,039 & 0,04 & 0,039 & 0,039 & 0,049 & 0,055 & 0,032 & 0,035 & 0,023 & 0,03 & 0,039 & 0,043 \\
\hline Chacal & 0,006 & 0,007 & 0 & 0 & 0 & 0 & 0,001 & 0,003 & 0 & 0 & 0,001 & 0,001 & 0,001 & 0,002 \\
\hline Civette & 0 & 0 & 0 & 0 & 0 & 0 & 0,001 & 0,004 & 0 & 0 & 0 & 0 & 0,000 & 0,001 \\
\hline Koba & 0,101 & 0,599 & 0,102 & 0,533 & 0,139 & 0,667 & 0,12 & 0,781 & 0,105 & 0,401 & 0,081 & 0,486 & 0,108 & 0,578 \\
\hline Cobe de buffon & 0,001 & 0,004 & 0,004 & 0,017 & 0,009 & 0,039 & 0,004 & 0,016 & 0,003 & 0,006 & 0,004 & 0,022 & 0,004 & 0,017 \\
\hline Eléphant & 0,03 & 0,299 & 0,038 & 0,354 & 0,032 & 0,211 & 0,069 & 0,489 & 0,042 & 0,247 & 0,04 & 0,232 & 0,042 & 0,305 \\
\hline Guib harnaché & 0,069 & 0,074 & 0,061 & 0,074 & 0,052 & 0,059 & 0,051 & 0,068 & 0,022 & 0,027 & 0,049 & 0,062 & 0,051 & 0,061 \\
\hline Mangouste & 0 & 0 & 0 & 0 & 0 & 0 & 0,001 & 0,017 & 0 & 0 & 0 & 0 & 0,000 & 0,003 \\
\hline Ourébi & 0,023 & 0,035 & 0,039 & 0,053 & 0,033 & 0,055 & 0,046 & 0,069 & 0,033 & 0,059 & 0,025 & 0,035 & 0,033 & 0,051 \\
\hline Patas & 0,012 & 0,038 & 0,014 & 0,071 & 0,004 & 0,004 & 0,013 & 0,042 & 0,007 & 0,016 & 0,014 & 0,027 & 0,011 & 0,033 \\
\hline Phacochère & 0,127 & 0,471 & 0,124 & 0,42 & 0,128 & 0,397 & 0,089 & 0,28 & 0,062 & 0,214 & 0,077 & 0,251 & 0,101 & 0,339 \\
\hline Redunca & 0,004 & 0,006 & 0,013 & 0,014 & 0,003 & 0,004 & 0,003 & 0,006 & 0,001 & 0,004 & 0,004 & 0,009 & 0,005 & 0,007 \\
\hline Vervet & 0,001 & 0,003 & 0,01 & 0,02 & 0,007 & 0,033 & 0,009 & 0,025 & 0,004 & 0,009 & 0,003 & 0,01 & 0,006 & 0,017 \\
\hline Waterbuck & 0,033 & 0,165 & 0,075 & 0,385 & 0,051 & 0,28 & 0,055 & & 0,043 & 0,136 & 0,052 & 0,263 & 0,052 & 0,205 \\
\hline
\end{tabular}


Tableau 2: Test de distribution spatiale avec les valeurs d'Indice de Blackman (1942).

\begin{tabular}{lccc}
\hline Paramètres & \multicolumn{3}{c}{ Périodes } \\
\cline { 2 - 4 } & $\mathbf{p 1}$ & $\mathbf{p 2}$ & $\mathbf{p 3}$ \\
\hline Moyenne & 4,57 & 4,26 & 4,21 \\
Variance & 33,97 & 25,68 & 24,63 \\
IB & 7,44 & 6,02 & 5,85 \\
Distribution & Agrégative & Agrégative & Agrégative \\
\hline
\end{tabular}

Tableau 3: Répartition spatiale globale de la faune.

\begin{tabular}{ccccccc}
\hline & \multicolumn{6}{c}{ Densités $\left(\mathbf{k m}^{\mathbf{2}}\right)$} \\
\cline { 2 - 7 } Périodes & $\mathbf{d 2}$ & $\mathbf{d 3}$ & $\mathbf{d 4}$ & $\mathbf{d 5}$ & TOTAL & \% \\
\hline P1 & 252,97 & 113,49 & 51,62 & 11,98 & 430,06 & 44,14 \\
P2 & 110,19 & 123,62 & 69,13 & 18,05 & 320,99 & 32,94 \\
P3 & 228,14 & 14,93 & 6,76 & 4,8 & 254,63 & 26,13 \\
\hline
\end{tabular}

Les effectifs de phacochère et de guib harnaché ont beaucoup varié dans le temps. Pour chacune des deux espèces, les plus faibles effectifs ont été enregistrés entre 1993 à 1997 d'après Ouédraogo (2005), "période noire" connue par le Ranch pendant laquelle, il a été enregistré la recrudescence du braconnage puis la dégradation de plusieurs aménagements. Une analyse sur les six inventaires de 2004 à 2009 montre une tendance à la baisse des effectifs des deux espèces.

$\mathrm{Au}$ regard de l'évolution tendancielle des populations de bubale, de koba, du phacochère et du waterbuck, et sous réserve d'études du maintien de cette tendance dans les recensements ultérieurs, on pourrait conclure partiellement que ces espèces peuvent continuer à être exploitées en quota de chasse sans souci d'entamer le capital faunique. Par contre, il serait souhaitable d'arrêter l'octroie de quota du céphalophe de grimm et du buffle pour une période permettant le repeuplement de leurs populations.
Le constat général qui découle de cette étude est qu'on note une instabilité des effectifs des différentes espèces animales observées au RGN depuis 1985 à 2009. Cette situation résulte des stratégies de gestion mises en place par l'administration de Ranch, de la dynamique évolutive naturelle des populations animales en liaison avec la disponibilité des ressources vitales, puis des pressions anthropiques diverses. De plus, du fait de l'existence d'autres aires protégées adjacentes, les instabilités d'effectif pourraient s'expliquer aussi par d'éventuels mouvements entre la forêt classée Kaboré-Tambi, la forêt classée de Sissili et les onze (11) zones villageoises de chasse adjacentes. Les échanges non structurés avec les riverains ont témoigné de ces mouvements d'animaux entre les aires protégées connexes. Ces observations confirment les travaux de Ouédraogo (2005) qui a démontré le mouvement de certaines espèces animales entre les terroirs villageois et le Ranch. 
Effet des indices anthropiques sur la distribution de la faune

La faune durant toutes les périodes allant de 2004 à 2009 a présenté une distribution agrégative. Ce qui témoigne de son retrait vers la partie centrale la plus fournies en eau et où règne une relative quiétude. Ce même comportement a été observé dans le Parc National de la Bénoué au Cameroun (Tsakem, 2006). En six (06) ans, la faune a connu une réduction de son domaine vital de $18 \%$. De façon générale, les animaux sont moins observés dans les régions de Sud, Est et Nord. Ces parties du RGN sont reconnues comme étant les zones de prédilection de braconnage et de pâturage illégal des bœufs. Cette situation est habituelle Cornelis (2000). Les noyaux de concentration observés loin des points d'eau montrent qu'il existe aussi d'autres facteurs environnementaux qui déterminent la présence de la faune. Ainsi, les espèces les moins dépendantes de l'eau, peuvent vivre bien loin des points d'eau et exploitent les habitats autres que les galeries, tandis que celles dépendantes de l'eau telles que le guib harnaché, le phacochère et le waterbuck se retrouvent dans les galeries et plaines inondables. Le comportement spatial de la faune révèle les menaces humaines en présence.

Ces résultats doivent permettre aux gestionnaires de tirer des leçons afin de renforcer le système de sécurisation du ranch et de ses ressources fauniques. Le travail devra continuer aussi bien dans le sens de l'implication des communautés locales que dans la surveillance. Une stratégie d'implication et de gestion transfrontalière devra être pensée pour impliquer les communautés sœurs de l'autre côté de la frontière ghanéenne, car elles sont les principales sources des pressions de braconnage.

\section{Conclusion}

Les estimations des effectifs et des densités par la méthode de "Distance sampling" ont été seulement effectuées pour le bubale, le céphalophe de grimm, le koba, le guib harnaché, le phacochère et le waterbuck. De même, la méthode des Indices Kilométrique d'Abondance a permis de révéler l'importance des éléphants au RGN. Les effectifs des espèces telles que le buffle et l'éléphant non pas été estimés en raison de l'inadéquation de la méthode de "Distance sampling" pour les espèces à faible densité. Le céphalophe de grimm, tout comme le buffle, se raréfie et nécessite des conditions pouvant favoriser leur repeuplement.

De façon générale, il a été noté une fluctuation des populations animales dans le temps et dans l'espace avec parfois de grandes variations qui sont surtout dues aux facteurs écologiques (biotiques et abiotiques), au biais induits par les méthodes et mouvements des populations. L'analyse de la distribution spatiale a révélé que l'eau et les pressions anthropiques influencent fortement la distribution de la faune au RGN. Cette situation interpelle non seulement l'administration du RGN, mais tous les partenaires à repenser une stratégie plus appropriée de sécurisation de l'aire protégée. Ceci permettrait à la faune d'exploiter au mieux son espace, et au Ranch de continuer à jouer son rôle de modèle d'aire protégée en Afrique de l'Ouest.

\section{REFERENCES}

Bouché P. 2004. Analyse comparée de différentes méthodes de comptage de la faune utilisées en Afrique de l'Ouest, West African Wildlife Services.

Brian RM. 2007. Comparison of Programs for Fixed Kernel Home Range Analysis. Rubenstein School of Environment and Natural Resources, University of Vermont. http://www.wildlife.org/wg/ gis/newsletter/jun06/hrcompar.htm.

Brimicombe AJ. 2007. Detecting clusters in spatially repetitive point event data sets. Cybergeo : European Journal of 
Geography. http://cybergeo.revues.org/ 8462 ; DOI : 10.4000/cybergeo.8462.

Buckland ST, Anderson DR, Burnham KP, Laake JL, Borchers D, Thomas L. 2004. Advanced Distance Sampling. Oxford University Press: Oxford.

Cornelis D. 2000. Analyse du monitoring écologique et cynégétique des populations des principaux ongulés au Ranch de Gibier de Nazinga (Burkina Faso). Mémoire de DEA, Faculté universitaire des Sciences agronomiques de Gembloux, Gembloux, Belgique, p.113.

Dekker AJFM. 1985. Carte de paysage de la region du Ranch de Gibier de Nazinga, Burkina Faso. PNUD/FAO : DP/BKF/82/008.

Delvingt W, Vermeulen C. 2007. Nazinga. Les Presses Agronomiques de Gembloux: Gembloux, Belgique.

Dibloni OT. 2003. Dynamique des populations d'hippotragues (Hippotragus equinus) et de bubales (Alcelaphus buselaphus) au Ranch de gibier de Nazinga. Mémoire de fin d'étude pour l'obtention du Diplôme d'Etude Approfondie en Sciences Agronomiques et Ingénierie biologique, Faculté universitaire des sciences agronomiques de Gembloux, Belgique, p.78.

Doumengue C, Garcia Yuste J-E, Gartlans S, Langrand O, Ndinga A. 2001. Conservation de la biodiversité forestière en Afrique centrale atlantique : le réseau d'aires protégées est-il adéquat ? Bois et Forêts des Tropiques, 268(2): 5-27.

Drouet-Hoguet N. 2007. Influence des activités anthropogéniques sur le régime alimentaire et la réponse numérique de l'hyène tachetée en savane arborée dystrophique dominée par l'éléphant. Thèse présentée pour l'obtention du Diplôme de Doctorat, UMR CNRS 5558. Laboratoire de Biométrie et
Biologie Evolutive, Université Claude Bernard Lyon I, France, p. 152.

Laake JL, Buckland ST, Anderson DR Burnham KP. 1994. Distance User's Guide V2.1. Colorado Cooperative Fish \& Wildlife Research Unit, University Colorado: Colorado State, Fort Collins, CO.

Laver PV. 2005. Kernel Home Range Estimation for ArcGis, Using VBA and Arc Objects. Use Manual (Beta v2. Adobe). Department of Fisheries and Wildlife Sciences, Virginia Tech, 149 Cheatham Hall, Blacksburg: Virginia, USA.

O’Donoghue M. 1986. Recensements à pied des grands mammifères au Projet pilote pour l'utilisation rationnelle de la faune à Nazinga. Rapport spécial, Séries C: $\mathrm{n}^{\circ}$ 13. Ranch de Gibier de Nazinga, Burkina-Faso.

Ouédraogo M. 2005. Régulation de la dynamique des populations de buffles (Syncerus caffer Sparrman) et de Waterbuck (Kobus ellipsiprymnus Ogilby) et moyens de gestion à mettre en œuvre pour préserver l'équilibre des communautés végétales dans le ranch de Nazinga (Burkina-Faso). Thèse de Doctorat en Agronomiques, et Ingénierie Biologique, Faculté Universitaire des Sciences Agronomiques de Gembloux, Belgique, p. 271.

PNGFAP. 2006. Composante : Gestion de La Faune et des Aires de Protection Fauniques. Ministère de l'Environnement et du cadre de vie, Burkina-Faso, p. 94 .

Sivakumar MVK, Gnoumou F. 1987. Agroclimatologie de l'Afrique de l'Ouest: le Burkina-Faso. Bulletin d'information $n^{\circ} 23$. International Crops Research Institute for the semi-Arid Tropics Patancheru, Andhra Pradesh 502 324, Inde. 192p. 
SOFRECO. 2003. Etude d'optimisation de la diversité biologique et de la rentabilité économique du ranch de faune de Nazinga. Rapport à mi-parcours, p. 108.

Tsakem SC. 2006. Contribution à l'aménagement du Parc National de la Bénoué et au développement rural des zones d'intérêt cynégétique à cogestion $\left(\mathrm{N}^{\circ} 1\right.$ et 4$)$ au Nord-Cameroun. Mémoire de fin d'étude, Université de Liège, Belgique.

Vermeulen C, Lamon A, Kabore B, Lankoande A. 2007. Le foncier en pratique : la délimitation de zones villageoises d'intérêt cynégétique en périphérie du parc du W. Vertigo Hors Série, 4(8): 1-9.
World Bank. 2008. Report on a global environment facility grant in the amount of us\$ 7.5 million equivalent to BurkinaFaso for a partnership for natural ecosystem management project. Sustainable development department environment department Africa region, p.60.

Worton BJ. 1989. Kernel methods for estimating the utilization distribution in home-range studies. Ecology, 80: 164168.

Yaméogo UG. 2005. Le feu, un outil d'ingénierie écologique au Ranch de Gibier de Nazinga au Burkina-Faso. Thèse de doctorat, Université d'Orléans, France. 